\title{
Functional Independence Measure in Iran: A Confirmatory Factor Analysis and Evaluation of Ceiling and Floor Effects in Traumatic Brain Injury Patients
}

\author{
Sajjad Rezaei, ${ }^{1, *}$ Anoush DehnadiMoghadam, ${ }^{1,2}$ Naeima Khodadadi, ${ }^{1,3}$ and PardisRahmatpour ${ }^{3}$ \\ ${ }_{2}^{1}$ Guilan Road Trauma Research Center, Guilan University of Medical Sciences, Rasht, IR Iran \\ ${ }^{2}$ Department of Anesthesiology and Intensive Care Unit, Guilan Road Trauma Research Center, Guilan University of Medical Sciences, Rasht, IR Iran \\ ${ }^{3}$ Department of Nursing, Faculty of Nursing and Midwifery, Guilan University of Medical Sciences, Rasht, IR Iran \\ ${ }^{*}$ Corresponding author: Sajjad Rezaei, Guilan Road Trauma Research Center, Guilan University of Medical Sciences, Rasht, IR Iran. Tel: +98-9113390785, Fax: +98-1333338373, \\ E-mail: Rezaei_psy@hotmail.com
}

Received 2014 December 26; Revised 2015 March 5; Accepted 2015 June 30.

\begin{abstract}
Background: The functional independence measure (FIM) is one of the most important assessment instruments for motor and cognitive dependence in rehabilitation medicine; however, there is little data about its confirmatory factor analysis (CFA) and ceiling/floor effects from other countries and also in Iranian patients.

Objectives: The aim of this study was to evaluate a two-factor model (motor and cognitive independence as latent variables) and ceiling/ floor effects for FIM in Iranian patients with traumatic brain injuries (TBI).

Patients and Methods: In this cross-sectional study, 185 subacute TBI patients were selected from emergency and neurosurgery departments of Poursina Hospital (the largest trauma hospital in northern Iran, Rasht) using the consecutive sampling method and were assessed for functional independence.

Results: The results of this study showed that the floor effect was not observed; however, ceiling effects were observed for the FIM total score and its subscales. The confirmatory factor analysis showed that the chi-square/df ratio was 2.8 for the two-factor structure and the fit indices for this structural model including root mean square error of approximation (RMSEA) $=0.099$, normed fit index $(\mathrm{NFI})=0.96$, tucker lewis index $(\mathrm{TLI})=0.97$, comparative fit index $(\mathrm{CFI})=0.97$ were close to standard indices.

Conclusions: Although ceiling effects should be considered for rehabilitation targets, the two-factor model of FIM (motor and cognitive independence) has an eligible fitness for Iranian patients with TBI.
\end{abstract}

Keywords: Traumatic Brain Injury, Confirmatory Factor Analysis, Disability Evaluation

\section{Background}

Traumatic brain injury (TBI) is one of the leading causes of death and long-term disability, especially in young adults (1). Traumatic brain injury survivors often suffer from high levels of physical disabilities, and cognitive and behavioral disorders, which severely affect their quality of life (2). These patients are often referred to rehabilitation centers so as to reduce morbidity, to improve functional outcomes, such as daily activities and to facilitate the transition from medical centers to home environment and access to self-care (3).

Lifetime costs of care for TBI victims are high and the influence of an effective treatment for this devastating disorder is economically remarkable not only for patient's family but also for society and health care system (4). Thus, the main goal of new treatment and interventions is to reduce disability of TBI patients and it is clear that clinicians, researchers and health system planners need instruments by which they can evaluate the effectiveness of care interventions in patients who may be sensitive to a variety of deficits, which typically create functional disabilities in TBI patients. One of the most widely accepted tools for measuring outcome and disability in hospitalized patients and those referred to rehabilitation centers is functional independence measure (FIM). The functional independence measure is the most useful instrument for measuring the patients' performance and effectiveness of rehabilitation program, which evaluates the daily activities extensively covering cognitive and motor domains (5).

This instrument was devised to solve the problem of long-term absence of a single method of disability assessment and thus creating unified data based on rehabilitation outcome. The functional independence measure is a product of studies by American Association

Copyright (c) 2015, Kashan University of Medical Sciences. This is an open-access article distributed under the terms of the Creative Commons Attribution-NonCommercial 4.0 International License (http://creativecommons.org/licenses/by-nc/4.0/) which permits copy and redistribute the material just in noncommercial usages, provided the original work is properly cited. 
of Rehabilitation Medicine and Physical Medicine and Rehabilitation Academy of America (6). This scale has 18 items (13 motor, 5 cognitive). For its grading, a multidisciplinary team observed the patient's function at 18 activities and evaluated the ability of patient for each activity from 1 to 7 , where 7 indicates complete independence or normal functioning score and 1 indicates complete dependence or needing help with all activities of daily living. The difference between the scores of total rated 18 items ranged from 18 (complete dependence) to 126 (complete independence). The functional independence measure can be used successfully in rehabilitation centers, hospitals, clinics, nursing homes and private care centers during the admission or patients' discharge $(6,7)$.

In instruments associated with outcome measurement in rehabilitation, identifying items with ceiling and floor effects is important; such items indicate activities that have little variability among patients. On FIM, Stineman and colleagues (8) suggested that such items could not be expected to contribute to the properties of the FIM, nor did they enhance the predictive value of its subscales. Kohler and colleagues (9) also examined patients who were transferred to other departments from a rehabilitation center and found that all cognitive FIM items and "eating" item in the motor subscale had a ceiling effect.

Traumatic brain injuries caused by traffic accidents have increased remarkably in Iran in recent years (10). However, regarding the developments in emergency measures and treatment of neurosurgery, there has been an increase in the survival rate of TBI victims. Therefore, introducing the Persian version of an instrument seems to be necessary for rehabilitation interventions and examining the outcomes. Cognitive and motor deficits after TBI are the two more common outcomes $(11,12)$ and two key objectives for rehabilitation $(13,14)$. Although conducted studies to examine the FIM factor structure showed that this scale is multi-factorial in nature (15), factor analysis studies concluded that FIM had a two-dimensional construct, which was determined by cognitive and motor domains (8). Thus, FIM can well measure motor and cognitive deficits in rehabilitation settings. Though, all studies related to factor structure consistently found that FIM cognitive subscale has one-dimensional construct, and any other adjunct factors, were related to motor FIM (16).

\section{Objectives}

This study aimed to evaluate the ceiling and floor effects for FIM, and to determine the dimensionality of FIM with two motor and cognitive independence factors in TBI patients referred to Poursina Hospital in Rasht city, Iran.

\section{Patients and Methods}

\subsection{Procedure}

In this cross-sectional study, 185 patients were selected from the emergency and neurosurgery departments of Poursina Hospital in Rasht City (the largest trauma hospital in northern Iran) using the consecutive sampling from 2011 to 2012. Based on the diagnosis of a neurosurgeon or emergency medicine physician, the FIM was conducted by two trained senior nurses.

\subsection{Training of the Raters and Translation of the Functional Independence Measure}

Two trained senior nurses referred to emergency and neurosurgery departments at the hospital for daily sampling. Before sampling, each of these nurses participated in a 5-session training course at least for 15 hours about the effects of TBI on cognition and motor skill of patients, reliability and validity of tests, familiarization and conducting the task and scoring and interpreting FIM scores in Guilan Road Trauma Research Center of Guilan University of Medical Sciences. To translate FIM, first its English version was rendered into Persian by two researchers and then separately translated from Persian into English by two English translators. Then, the four translated versions were compiled and the final version of Persian FIM was designed. To examine the content validity and applicability of the Persian version for TBI patients, two neurologists, four nurse researchers, one neurosurgeon and one anesthesiologist at Guilan University of Medical Sciences were asked for their comments. After that, the original Persian FIM was prepared.

\subsection{Eligibility and Recruitment}

The inclusion criteria, according to a neurosurgeon diagnosis, were as follows: age $\geq 16$ years, Glasgow Coma Scale $(\mathrm{GCS})<15$, focal or diffuse damage to brain tissue caused by an external mechanical force, loss of consciousness (LOC) for more than 1 minute, length of posttraumatic amnesia (PTA) over 20 minutes, radiographic or computerized tomography (CT) scan findings showing TBI (skull fractures, intracranial bleeding or brain acute abnormalities), and hospitalization of less than one week. Exclusion criteria were patients with clinical or radiological findings manifesting spinal cord injury, any neurological disease before TBI or brain injury with nontraumatic origin (brain tumors, stroke, aneurism and other cerebrovascular accidents), patients with vegetative state or severe loss of consciousness so that they were unable to answer, movement or balance disorders, arthritis, knee and joint 
Rezaei S et al.

fractures before TBI, patients who were not satisfied to enter the study for any reason and those who had hospitalized for more than one week. This study was approved by the research ethics committee of Poursina Hospital in Rasht. At the beginning of evaluating the TBI patients, the participants were informed about the reasons and procedure of the study. They were assured that their information would be confidential and they can withdraw from the study at any time. First, an informed consent was obtained from all participants or their legal caregivers and assessments were then performed.

\subsection{Statistical Analysis}

In this study, to describe the data, descriptive statistical parameters such as frequency, percentage, Mean and Standard Deviation were used. Ceiling and floor effects (a percentage of the sample, which takes the lowest and the highest scores) indicate the extent to which score clusters are located at the bottom or top of the scale range. Ideally, the observed scores of a scale should be placed in the whole range of the scale. The average score should be close to the midpoint of the scale, having the small ceiling and floor effects. McHorney and Tarlov (17) have suggested that ceiling and floor effects should be lower than 15\%. All the statistical analyses were performed by a significance level of less than $\mathrm{P}<0.05$ using the PASW® software (version 22).

Furthermore, to study the current two-factor structure of FIM (motor and cognitive), confirmatory factor analysis (CFA) was used with maximum likelihood by Amos software (version 21.0). Confirmatory factor analysis seeks to answer the question that assumed or anticipated relationships among variables, "to what extent the observed relationships are consistent with real data?" If two correlation matrices (the assumed correlation matrix and the proposed correlation matrix to be derived from actual data) are consistent with each other, the proposed model would be a valid explanation for the hypothesized relationships (18). Structural equation modeling approach was used to determine the sample size for CFA, which was proposed by Kline (19). A sample of 180 was acceptable at 10:1 ratio subjects for each 1 item). Given that the 18 items of FIM were included in this analysis, thus 180 patients are needed, 185 patients were sampled until complete sample size (but 5 questionnaires was incomplete).

\section{Results}

\subsection{Demographic Data of the Participants}

In the subacute phase, 185 TBI patients in the age range of 16 to 85 years (mean age $37.45 \pm 17.42$ ) were participated in the study. These patients were hospitalized in emergency department and neurosurgery section for approximately 48 and 96 hours (2 and 4 days), respectively. The average education level of these patients was $7.31 \pm 4.49$ years in a range between 0 to 16 years. Table 1 shows the findings of demographic and clinical variables of the TBI patients.

\subsection{Confirmatory Factor Analysis}

A confirmatory factor analysis was performed on covariance matrix of motor and cognitive FIM items. Model parameters were estimated using maximum likelihood. The reference model tested in this study included 18 items, which were loaded on two FIM latent factors. In this model, all covariances between the measurement errors of observed variables (indicator) were fixed. The results were shown in Table 2. As fit indices indicated, this hypothesized model did not fit the data well (reference model). With more investigation of FIM items found that some of them were similar and particularly representative of self-care skills (for example, items 2 to 6). By investigating Amos modification indices, specific error covariance terms were freed consecutively; which means that after freeing the error covariance between certain items, the fit indices were examined to answer this question: "Have they improved the model?"

As seen in Table 2, by freeing 12 error covariance terms between items, the model's fitness was significantly improved. Through sequentially incorporating error covariance terms inside the model, a consistent fitness was obtained conformed to a two-factor structure for FIM (see Model 12 in Table 2). Comparing fit indices between the reference model and last modified model (chi-square/df, RMSEA, NFI, TLI, CFI, AIC) reflects that the modified two-factor model has the best overall fitness to the data in FIM motor and cognitive subscales in Iranian TBI patients (chi-square/df $=2.8$, RMSEA $=0.099, \mathrm{NFI}=0.96, \mathrm{TLI}=0.97, \mathrm{CFI}=0.97$ ). The final model of the FIM two-factor analysis is presented in Figure 1.

\subsection{Floor and Ceiling Effect}

Table 3 shows the results of the Mean, Standard Deviation, and floor and ceiling effects for each item. Also, total FIM and motor and cognitive subscales are presented.

As seen in Table 3, in total score, subscales and each of FIM items, the floor effect was not observed; however, the ceiling effect in the total score, subscales (motor and cognitive) and the items of eating, bowl, bladder, comprehension, expression, social interaction, problem solving and memory were observed. 
Rezaei S et al.

\begin{tabular}{|c|c|}
\hline Variables & Values $^{b}$ \\
\hline \multicolumn{2}{|l|}{ Gender } \\
\hline Men & $171(92.4)$ \\
\hline women & $14(7.6)$ \\
\hline \multicolumn{2}{|l|}{ Marital status } \\
\hline Single & $66(35.7)$ \\
\hline Married & $118(63.8)$ \\
\hline Unknown & $1(0.5)$ \\
\hline \multicolumn{2}{|l|}{ Admission GCS } \\
\hline 8 or less & $19(10.3)$ \\
\hline 9 to 12 & $21(11.3)$ \\
\hline 13 to 15 & $145(78.4)$ \\
\hline \multicolumn{2}{|l|}{ Discharge GCS } \\
\hline 8 or less & $4(2.2)$ \\
\hline 9 to 12 & $9(4.8)$ \\
\hline 13 to 15 & $173(93)$ \\
\hline \multicolumn{2}{|l|}{ Cause of TBI } \\
\hline Accident & $27(14.7)$ \\
\hline Motorcycle & $91(49.2)$ \\
\hline Pedestrian & $15(8.1)$ \\
\hline Fall & $16(8.6)$ \\
\hline Violence & $5(2.7)$ \\
\hline Hit the object & $11(5.9)$ \\
\hline Backfall & $18(9.7)$ \\
\hline Cycling & $1(0.5)$ \\
\hline Unknown & $1(0.5)$ \\
\hline \multicolumn{2}{|l|}{ Skull fracture } \\
\hline No & $118(63.8)$ \\
\hline Linear & $47(25.4)$ \\
\hline Depressed & $16(8.6)$ \\
\hline Basilar & $2(1.1)$ \\
\hline Synthetic & $1(0.5)$ \\
\hline Unknown & $1(0.5)$ \\
\hline \multicolumn{2}{|l|}{ Hemisphere lesion } \\
\hline No & $20(10.8)$ \\
\hline Left & $59(31.9)$ \\
\hline Right & $78(42.2)$ \\
\hline Bilateral & $25(13.5)$ \\
\hline Unknown & $3(1.6)$ \\
\hline \multicolumn{2}{|l|}{ Lesion location } \\
\hline No & $17(9.2)$ \\
\hline Frontal & $49(26.5)$ \\
\hline Temporal & $54(29.2)$ \\
\hline Parietal & $15(8.1)$ \\
\hline The Series & $10(5.4)$ \\
\hline Multiple locations & $36(19.5)$ \\
\hline Unknown & $4(2.2)$ \\
\hline
\end{tabular}

${ }^{\mathrm{a}}$ Abbreviations: GCS, glasgow coma score; TBI, traumatic brain injury.

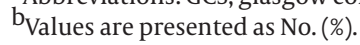


Rezaei S et al.

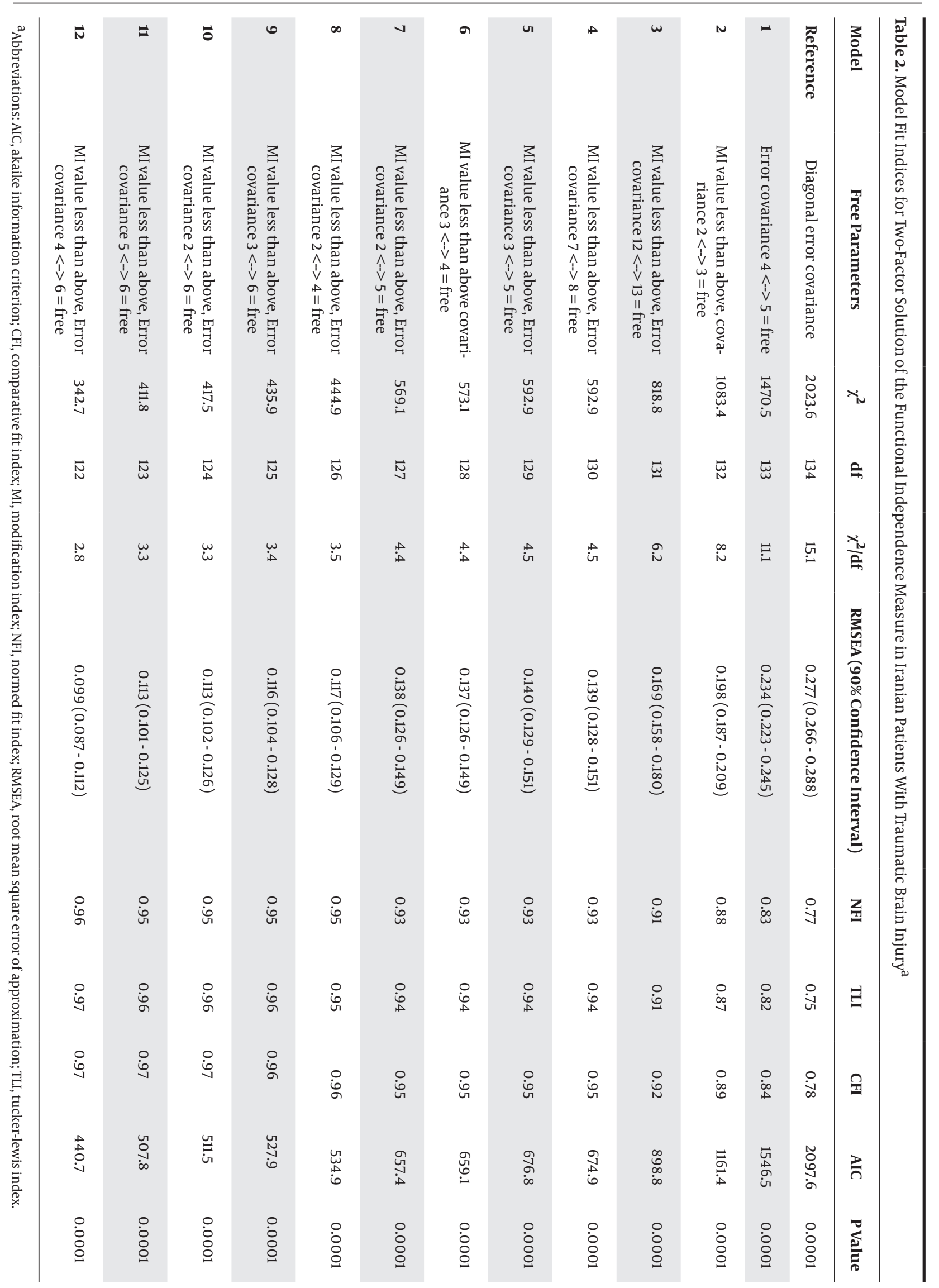




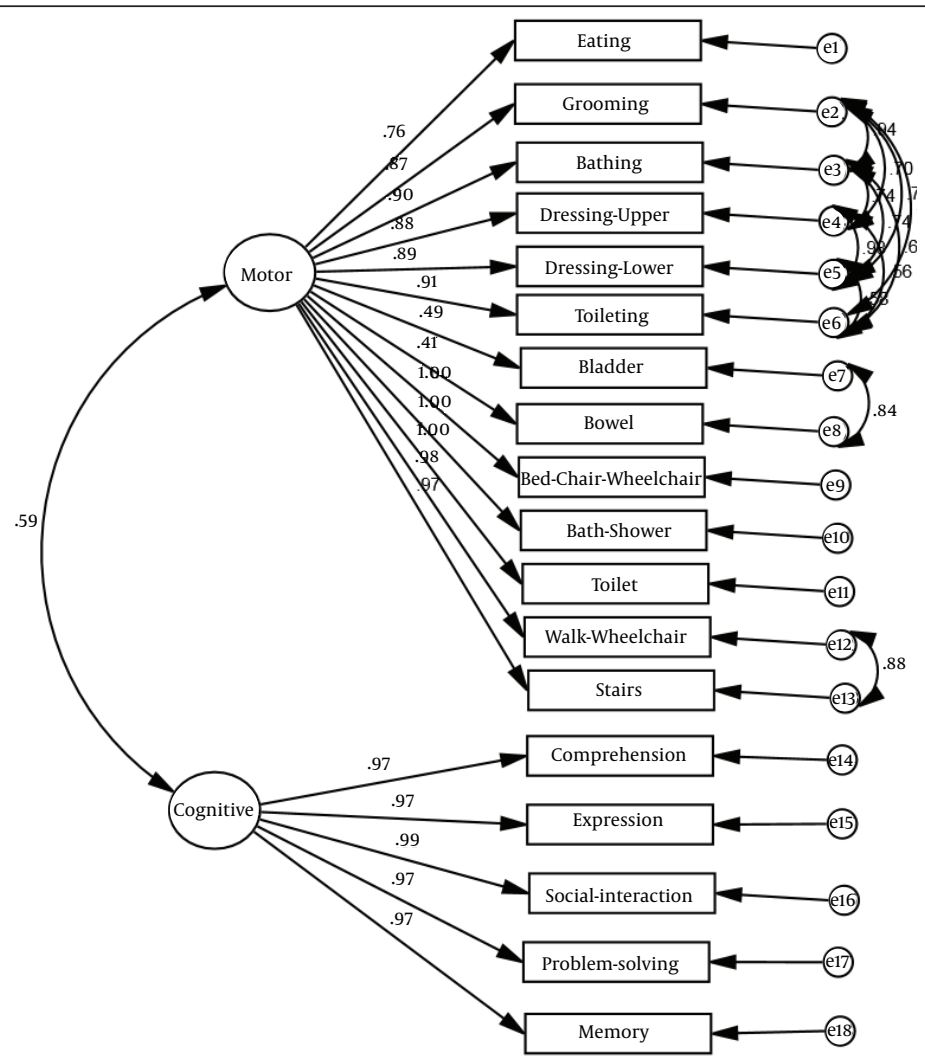

Figure 1. The Final Model for the Two-Factor Solution in Iranian Patients With Traumatic Brain Injury

\begin{tabular}{|c|c|c|c|}
\hline & Values $^{\mathbf{b}}$ & Floor Effect, \%c & Ceiling Effect, $\%{ }^{\mathrm{d}}$ \\
\hline \multicolumn{4}{|l|}{ Items } \\
\hline 1. Eating & $5.07 \pm 1.67$ & $6(3.24)$ & $59(31.9)$ \\
\hline 2. Grooming & $4.31 \pm 1.65$ & $10(5.40)$ & $26(14)$ \\
\hline 3. Bathing & $4.25 \pm 1.61$ & $10(5.40)$ & $22(11.9)$ \\
\hline 4. Dressing-Upper & $4.35 \pm 1.57$ & $9(4.86)$ & $25(13.5)$ \\
\hline 5. Dressing-Lower & $4.32 \pm 1.57$ & $9(4.86)$ & $23(12.4)$ \\
\hline 6. Toileting & $4.10 \pm 1.62$ & $10(5.40)$ & $23(12.4)$ \\
\hline 7. Bladder & $6.47 \pm 1.46$ & $10(5.40)$ & $152(82.2)$ \\
\hline 8. Bowel & $6.61 \pm 1.41$ & $9(4.86)$ & $170(91.9)$ \\
\hline 9. Bed/Chair/Wheelchair & $3.96 \pm 1.59$ & $10(5.40)$ & $19(10.3)$ \\
\hline 10. Bath/Shower & $3.96 \pm 1.58$ & $10(5.40)$ & $19(10.3)$ \\
\hline 11. Toilet & $3.95 \pm 1.58$ & $10(5.40)$ & $18(9.7)$ \\
\hline 12. Walk/Wheelchair & $3.88 \pm 1.60$ & $10(5.40)$ & $18(9.7)$ \\
\hline 13. Stairs & $3.85 \pm 1.61$ & $10(5.40)$ & $18(9.7)$ \\
\hline 14. Comprehension & $6.18 \pm 1.65$ & $9(4.86)$ & 131(70.8) \\
\hline 15. Expression & $6.15 \pm 1.76$ & $12(6.48)$ & 135(73.0) \\
\hline 16. Social interaction & $6.06 \pm 1.70$ & $9(4.86)$ & $123(66.5)$ \\
\hline 17. Problem solving & $5.89 \pm 1.81$ & $12(4.48)$ & $117(63.2)$ \\
\hline 18. Memory & $5.91 \pm 1.75$ & $11(5.94)$ & $109(58.9)$ \\
\hline FIM total & $89.35 \pm 24.97$ & $10(5.40)^{\mathrm{e}}$ & $39(21.1)^{\mathrm{e}}$ \\
\hline Motor subscale & $59.14 \pm 18.41$ & $9(4.86)^{f}$ & $32(17.3)^{f}$ \\
\hline Cognitive subscale & $30.20 \pm 8.50$ & $12(6.48)^{g}$ & $133(71.9)^{\mathrm{g}}$ \\
\hline $\begin{array}{l}a_{\text {Abbreviation: FIM, functional }} \\
\text { b Values are presented as mean } \\
c_{\text {Number of people with score }} \\
d_{\text {Number of people with score }} \\
e_{\text {Floor and ceiling effects of FIN }} \\
f_{\text {Floor and ceiling effects of mo }} \\
g_{\text {floor and ceiling effects of cog }}\end{array}$ & $\begin{array}{l}\text { and upper dist } \\
\text { lower and upp } \\
5 \% \text { lower and } \mathrm{u}\end{array}$ & $10(0.0)$ & \\
\hline
\end{tabular}




\section{Discussion}

The present study examined the two-factor pattern of FIM and its ceiling/floor effects in Iranian patients with TBI. The rate of accident is high in Iran and currently new attentions have been made to cognitive and motor outcomes in these victims that the most of whom have TBI (20). Thus, introducing and translating an efficient tool that might be used in evaluating motor and cognitive outcomes and rehabilitation in these patients are necessary.

In this study, while no floor effect was found in all items, total score and FIM subscales, but in line with the results of Kohler and colleagues (9) many patients showed a clear ceiling effect in five FIM cognitive items and as well as eating, bowel and bladder control items and FIM total score and its subscales (Table 3 ). Items with ceiling effects are generally representative of the activities in which every patient has a good functioning closely in a category of injury, and thus rehabilitation purposes may be less affected by the items. In this case, Cohen and Marino (21) have suggested that, compared with outpatient settings, FIM may be useful in inpatient settings.

Different factor structures for FIM has been introduced (16), but these tools are often used for the assessment of motor and cognitive outcomes and rehabilitation intervention $(20,22)$, especially in TBI patients $(20,23)$. According to the previous studies, the reference model in this study is consistent with the classification of motor (13 items) and cognitive (5 items) parts underwent CFA. However, the fit indices were far from acceptable standards (chi-square/df $=15.1$, RMSEA $=0.277, \mathrm{NFI}=0.77$, TLI $=0.75, \mathrm{CFI}=0.78)$. This finding was not unexpected. Typically, in CFA, the initial model cannot achieve a good fit (24), thus through a discovery process in accordance with the modification indices freed the 12 error covariance terms between FIM items (particularly items 2 to 6) (25) and thereupon the final model showed a good recovery (chi-square $/ \mathrm{df}=2.8, \mathrm{RMSEA}=0.099, \mathrm{NFI}=0.96, \mathrm{TLI}=0.97$, $\mathrm{CFI}=0.97)$. Comparing the results of the research literature showed that so far no study has performed CFA on 18-item version of FIM in adults or TBI patient population. However, Park et al. (26) investigated the confirmatory model of WeeFIM in Korean children with cerebral palsy and concluded that the one-factor model (18 items WeeFIM) and the two-factor (13 motor items and 5 cognitive items) had not enough fitness, but the three-factor WeeFIM model, including the factor of 6 self-care items (i.e. eating, grooming, dressing upper, dressing lower, bathing, and toileting), the factor of 7-item motor (i.e. bladder and bowel management, bed/chair/wheelchair, toilet, tub/shower, walk/wheelchair, and stairs) and the factor of 5-items cognitive (the 5 original items of cognitive domain) had fitness with WeeFIM data of cerebral palsy children. As the content of covariate errors in this study and the results of Park et al. study (26) show, it can be withdrawn, "the latent self-care factor" which is nested within the FIM motor domain has tendency to appear in Iranian TBI patients. Results of factor analysis in this study repeat the motor and cognitive dimensions of FIM, which were first introduced by Linacre et al. (27) and Heinemann et al. (28). In World Health Organization, for the two-factor FIM as a state of physical, psychological and social well-being, there is a theoretical, clinical and statistical support.

As seen in Figure 1, the FIM motor dimension showed a strong correlation with cognitive dimension and excluding sphincter control items was significantly associated with other physical items (path coefficients $\geq 0.76$ ). In this model, bladder and bowel control items tend to show disproportionate levels of basic structure of motor FIM (path coefficients of 0.49 and 0.41 , respectively) for TBI patients. Along with this result, Kucukdeveci and colleagues (29) in a Rasch analysis in stroke and SCI patients also showed that bladder and bowel items breaks the integrity of the FIM motor subscale. This heterogeneity can be largely associated with the mismatch between the injury and the resulting disability. For example, some patients with severe TBI are able to control urine and feces, while patients with mild to moderate TBI can have serious inability to sphincter control. This does not necessarily indicate that the sphincter control items are poor, but as the values of GCS, and the various injuries associated with TBI in Table 1 can be due to the lack of homogeneous or dispersion in study population. In contrast to this inequity in motor items, FIM cognitive dimension was fully homogeneous and closely related to communication (comprehension and expression) and social cognition items (social interaction, problem-solving and memory) (Figure 1, all path coefficients $\geq 0.97$ ).

The results of this study highlight the need for motor and cognitive rehabilitation interventions to improve physical disability, quality of life and cognitive impairments in TBI patients. Totally, ceiling effects were observed in FIM total score and subscales; therefore, should be considered for rehabilitation targets. In addition, fit indices for the FIM two-factor model in CFA was not found in ideal criteria. However, during a modificational-exploratory process, the optimized two-factor model for Iranian patients with TBI was introduced. Although the two-dimensional measurement of motor and cognitive independence/disability in rehabilitation and outcome by FIM is common, in the meantime, considering a rival model of three-factor can be recommended for patients with TBI.

It must be noted that the results are not generalizable to patients with chronic phase TBI. Also, it should be cautioned in clinical decision making and interpreting the FIM results for subacute phase TBI, especially on items that have a ceiling effect. It can be suggested that this tool be used in future research for assessment of physical and cognitive rehabilitation effects in TBI patients in particular on chronic phase to reevaluate the applicability and its ceiling and floor effects. 


\section{Acknowledgments}

The authors wish to acknowledge the participation of clients and staff of the emergency department services and neurosurgery section at Poursina Hospital in Rasht city and they also would like to thank the colleagues in the clinical researches and development unit for their support, the Guilan University of Medical Sciences and Ms. Fatemeh Javadi for drafting the manuscript.

\section{Footnotes}

Authors' Contribution:Sajjad Rezaei: research design, supervision of the study, data analysis, and writing the article; Anoush Dehnadi-Moghadam: advisor and editor; Naeima Khodadadi: data collection, and evaluating of TBI patients; Pardis Rahmatpour: data collection, and evaluating of TBI patients.

Funding/Support:This research project was financially supported by Road Trauma Research Center in Guilan University of Medical Sciences.

\section{References}

1. Nichol AD, Higgins AM, Gabbe BJ, Murray LJ, Cooper DJ, Cam eron PA. Measuring functional and quality of life outcomes following major head injury: common scales and checklists. Injury. 2011;42(3):281-7. doi: 10.1016/j.injury.2010.11.047. [PubMed: 21145059]

2. Schretlen DJ, Shapiro AM. A quantitative review of the effects of traumatic brain injury on cognitive functioning. Int Rev Psychiatry. 2003;15(4):341-9. doi: 10.1080/09540260310001606728. [PubMed: 15276955]

3. Hukkelhoven CW, Steyerberg EW, Rampen AJ, Farace E, Habbema JD, Marshall LF, et al. Patient age and outcome following severe traumatic brain injury: an analysis of 5600 patients. J Neurosurg. 2003;99(4):666-73. doi: 10.3171/jns.2003.99.4.0666. [PubMed: 14567601]

4. McGarry LJ, Thompson D, Millham FH, Cowell L, Snyder PJ, Lenderking WR, et al. Outcomes and costs of acute treatment of traumatic brain injury. J Trauma. 2002;53(6):1152-9. doi: 10.1097/01. TA.0000025801.33552.71. [PubMed:12478043]

5. Shukla D, Devi BI, Agrawal A. Outcome measures for traumatic brain injury. Clin Neurol Neurosurg. 2011;113(6):435-41. doi: 10.1016/j.clineuro.2011.02.013. [PubMed: 21440363]

6. Granger CV. Guide for the Uniform Data Set for Medical Rehabilitation (including the FIM instrument) version 5.1.New York: Buffalo; 1997.

7. Hershkovitz A, Kalandariov Z, Hermush V, Weiss R, Brill S. Factors affecting short-term rehabilitation outcomes of disabled elderly patients with proximal hip fracture. Arch Phys Med Rehabil. 2007;88(7):916-21. doi: 10.1016/j.apmr.2007.03.029. [PubMed: 17601474]

8. Stineman MG, Shea JA, Jette A, Tassoni CJ, Ottenbacher KJ, Fiedler $\mathrm{R}$, et al. The Functional Independence Measure: tests of scaling assumptions, structure, and reliability across 20 diverse impairment categories. Arch Phys Med Rehabil. 1996;77(11):1101-8. [PubMed: 8931518]

9. Kohler F, Dickson H, Redmond H, Estell J, Connolly C. Agreement of functional independence measure item scores in patients transferred from one rehabilitation setting to another. Eur J Phys Rehabil Med. 2009;45(4):479-85. [PubMed: 20032905]

10. Cifu DX, Keyser-Marcus L, Lopez E, Wehman P, Kreutzer JS, Englander J, et al. Acute predictors of successful return to work1 year after traumatic brain injury: a multicenter analysis. Arch Phys
Med Rehabil.1997;78(2):125-31. [PubMed: 9041891]

11. Godbolt AK, Cancelliere C, Hincapie CA, Marras C, Boyle E, Kristman VL, et al. Systematic review of the risk of dementia and chronic cognitive impairment after mild traumatic brain injury: results of the International Collaboration on Mild Traumatic Brain Injury Prognosis. Arch Phys Med Rehabil. 2014;95(3 Suppl):S245-56. doi: 10.1016/j.apmr.2013.06.036. [PubMed: 24581910]

12. Breceda EY, Dromerick AW. Motor rehabilitation in stroke and traumatic brain injury: stimulating and intense. Curr Opin Neurol. 2013;26(6):595-601. doi: 10.1097/WCO.0000000000000024. [PubMed: 24141528]

13. Cernich AN, Kurtz SM, Mordecai KL, Ryan PB. Cognitive rehabilitation in traumatic brain injury. Curr Treat Options Neurol. 2010;12(5):412-23. doi: 10.1007/s11940-010-0085-6. [PubMed: 20842598]

14. Ravaud JF, Delcey M, Yelnik A. Construct validity of the functional independence measure (FIM): questioning the unidimensionality of the scale and the "value" of FIM scores. Scand J Rehabil Med. 1999;31(1):31-41. [PubMed:10230001]

15. Stineman MG, Jette A, Fiedler R, Granger C. Impairment-specific dimensions within the Functional Independence Measure. Arch Phys Med Rehabil.1997;78(6):636-43. [PubMed: 9196472]

16. Yousefzadeh Chabok S, Ramezani S, Kouchakinejad L, Saneei Z Epidemiology of pediatric head trauma in guilan. Arch Trauma Res. 2012;1(1):19-22. doi: 10.5812/atr.5289. [PubMed:24719836]

17. McHorney CA, Tarlov AR. Individual-patient monitoring in clinical practice: are available health status surveys adequate? Qual Life Res. 1995;4(4):293-307. [PubMed:7550178]

18. Byrne BM. Structural Equation Modeling With AMOS : Basic Concepts, Applications, and Programming. . Second Edition ed. New York: Taylor \& Francis; 2010.

19. Kline RB. Principles and practice of structural equation modeling. 3rd ed. New York: Guilford Press; 2010.

20. Rezaei S, Dehnadi Moghadam A, khodadadi N, Rahmatpour P, Salehpour G. Prediction of motor and cognitive outcome in acute traumatic brain injury based on length of hospital stay, Glasgow coma scale score (GCS), mental status and substance abuse: a case study of emergency and neurosurgery section in Rasht PourSina Hospital. J Iran Soci Anaesthesiology \& Intensive Care . 2013;82(2):24-35.

21. Cohen ME, Marino RJ. The tools of disability outcomes research functional status measures. Arch Phys Med Rehabil. 2000;81(12 Suppl 2):S21-9. [PubMed:11128901]

22. Smania N, Avesani R, Roncari L, Ianes P, Girardi P, Varalta V, et al. Factors predicting functional and cognitive recovery following severe traumatic, anoxic, and cerebrovascular brain damage. J Head Trauma Rehabil. 2013;28(2):131-40. doi: 10.1097| HTR.ob013e31823c0127. [PubMed: 22333677]

23. Perrin PB, Niemeier JP, Mougeot JL, Vannoy CH, Hirsch MA Watts JA, et al. Measures of injury severity and prediction of acute traumatic brain injury outcomes. J Head Trauma Rehabil. 2015;30(2):136-42. doi: 10.1097/HTR.0000000000000026. [PubMed: 24590151]

24. Meyers LS, Gamst G, Guarino AJ. Applied multivariate research: Design and interpretation. London,U.K.: New Delhi: Sage; 2006.

25. Bentler PM. Comparative fit indexes in structural models. Psychol Bull.1990;107(2):238-46. [PubMed:2320703]

26. Park EY, Kim WH, Choi YI. Factor analysis of the WeeFIM in children with spastic cerebral palsy. Disabil Rehabil. 2013;35(17):146671. doi: 10.3109/09638288.2012.737082. [PubMed: 23206258]

27. Linacre JM, Heinemann AW, Wright BD, Granger CV, Hamilton BB The structure and stability of the Functional Independence Measure. Arch Phys Med Rehabil. 1994;75(2):127-32. [PubMed: 8311667]

28. Heinemann AW, Hamilton B, Granger CV, Wright BD, Linacre IM Betts HB. Rating scale analysis of functional assessment measures. Chicago: Rehabilitation Institute of Chicago; 1991.

29. Kucukdeveci AA, Yavuzer G, Elhan AH, Sonel B, Tennant A. Adaptation of the Functional Independence Measure for use in Turkey. Clin Rehabil. 2001;15(3):311-9. [PubMed: 11386402] 